\title{
Novel nonreciprocal materials based on magnetic photonic crystals
}

\author{
A. Figotin and I. Vitebskiy \\ University of California at Irvine, \\ Irvine, CA 92697-3875, U.S.A.
}

\begin{abstract}
Magnetic photonic crystals are spatially periodic dielectric composites with at least one of the constitutive components being a magnetically polarized material. Magnetic polarization, either spontaneous or induced, is always associated with nonreciprocal circular birefringence (Faraday rotation), which can bring qualitatively new features to the electrodynamics of photonic crystals. If the geometry of the periodic array meets certain symmetry criterion, the electromagnetic properties of the composite appear similar to those of a hypothetical bianisotropic medium with gigantic linear magnetoelectric effect. In particular, such a photonic crystal can display sptrong spectral asymmetry, which implies that electromagnetic waves propagate from left to right significantly faster or slower than from right to left. The strong spectral asymmetry can result in the phenomenon of electromagnetic unidirectionality. A lossless unidirectional medium, being perfectly transmissive for electromagnetic wave of certain frequency, "freezes" the radiation of the same frequency propagating in the opposite direction. The frozen mode is a coherent Bloch wave with nearly zero group velocity and drastically enhanced amplitude. The phenomenon of electromagnetic unidirectionality is essentially nonreciprocal and unique to gyrotropic photonic crystals. Physical conditions for the phenomenon include (i) significant Faraday rotation in the magnetic component of the composite structure at the frequency range of interest and (ii) the proper spatial arangement of the constituents. Unidirectional photonic crystals can be very attractive for a variety of applications.
\end{abstract}

\section{ELECTROMAGNETICS OF NONRECIPROCAL PERIODIC MEDIA}

In spatially periodic media, such as photonic crystals, the electromagnetic eigenmodes can be represented in the Bloch form

$$
\Psi_{k}(\boldsymbol{r}+\boldsymbol{a})=\Psi_{k}(\boldsymbol{r}) \exp (i \boldsymbol{k} \cdot \boldsymbol{a})
$$

where $\boldsymbol{k}$ is the Bloch wave vector and $\boldsymbol{a}$ is a lattice translation. The correspondence $\omega(\boldsymbol{k})$ between the wave vector $\boldsymbol{k}$ and the frequency $\omega$ is referred to as the dispersion relation. In most cases, the dispersion relation is symmetric with respect to the wave vector

$$
\omega(\boldsymbol{k})=\omega(-k) .
$$

Usually, the relation (2) can be viewed as a direct consequence of time reversal and/or space inversion symmetry of the periodic array. Indeed, let $I$ and $R$ denote the space inversion and time reversal operations, respectively. Since either operation reverses the direction of the Bloch wave vector

$$
I k=-k, \quad R k=-k
$$


we can conclude that

$$
\text { if } R \in G \text { and/or } I \in G \text {, then } \omega(\boldsymbol{k})=\omega(-\boldsymbol{k}) \text { for any } \boldsymbol{k} \text {, }
$$

where $G$ is the magnetic symmetry group of the periodic array. All non-magnetic media support time reversal symmetry. In addition, most of the homogeneous and periodic heterogeneous structures are centrosymmetric. As a consequence, the overwhelming majority of homogeneous materials, as well as periodic composites, display perfectly symmetric dispersion relation.

Periodic medium can support asymmetric dispersion relation

$$
\omega(\boldsymbol{k}) \neq \omega(-k),
$$

only if

$$
R \notin G \text { and } I \notin G \text {. }
$$

The spectral asymmetry (4) implies that plane waves propagate in one direction faster or slower than in the opposite direction.

Even if the necessary condition (5) for spectral asymmetry is satisfied, there still might be some special directions of the wave vector $\boldsymbol{k}$ for which the dispersion relation $\omega(\boldsymbol{k})$ is symmetric. Specifically

$$
\omega(\boldsymbol{k})=\omega(-\boldsymbol{k}) \text { if } g \boldsymbol{k}=-\boldsymbol{k},
$$

where $g$ is a symmetry operation from the magnetic symmetry group $G$ of the periodic structure. Therefore, instead of (4), one can use stronger necessary condition for spectral asymmetry (see, for example, [1] and references therein)

$$
\omega(\boldsymbol{k}) \neq \omega(-k) \text { only if } g \boldsymbol{k} \neq-\boldsymbol{k} \text { for all } g \in G,
$$

Unlike (5), the stronger necessary condition (7) is sensitive to the direction of the Bloch wave vector $\boldsymbol{k}$. The criterion (7) will be used throughout the paper to find the proper geometry of magnetic photonic crystals, as well as directions of wave propagation exhibiting the desired property. Obviously, if the symmetry group $G$ includes time reversal and/or space inversion, the criterion (7) cannot be satisfied for any direction of $\boldsymbol{k}$.

Note that symmetry consideration based on the formulae (1) through (7) applies not only to electromagnetic waves, but also to any other linear excitations in periodic media, such as electrons, magnons, excitons, etc. But all the above questions go beyond the scope of our consideration. In this paper we are dealing exclusively with electromagnetic waves in photonic crystals.

Naturally occurring substances displaying electromagnetic spectral asymmetry (4) have been known for decades (see [2] and references therein). They constitute a special class of crystalline materials known as bianisotropic materials, or magnetoelectrics [2 - 4]. The electrodynamics of magnetoelectric media can be described by standard time-harmonic Maxwell equations 


$$
\nabla \times \boldsymbol{E}=\frac{i \omega}{c} \boldsymbol{B}, \quad \nabla \times \boldsymbol{H}=-\frac{i \omega}{c} \boldsymbol{D}
$$

with "bianisotropic" constitutive relations [3]

$$
\boldsymbol{D}=\boldsymbol{\varepsilon} \boldsymbol{E}+\chi \boldsymbol{H}, \quad \boldsymbol{B}=\mu \boldsymbol{H}+\chi^{T} \boldsymbol{E},
$$

where $\boldsymbol{\varepsilon}$ and $\boldsymbol{\mu}$ are the electric permittivity and magnetic permeability tensors, $\chi$ is the tensor of linear magnetoelectric response, and the subscript $T$ indicates matrix transposition. Unlike $\varepsilon$ and $\boldsymbol{\mu}$, the tensor $\chi$ is odd with respect to time reversal $R$ and space inversion $I$, implying that the linear magnetoelectric effect is ruled out in all nonmagnetic and/or centrosymmetric media [3]

$$
\chi \neq 0 \text {, only if } R \notin G, I \notin G \text {. }
$$

Comparison of the expressions (5) and (10) shows that necessary symmetry conditions of the linear magnetoelectric effect are similar to those of the spectral asymmetry. The problem with magnetoelectric crystals though is that the degree of electromagnetic spectral asymmetry in those substances turns out to be negligible $\left(10^{-4}\right.$ or less). The situation is further aggravated by complicated and often unpredictable domain structure of natural magnetoelectric materials. As a consequence, the remarkable properties of magnetoelectrics featuring electromagnetic spectral asymmetry (4) have not found any significant applications. The above problems can be avoided in magnetic photonic crystals, which are periodic arrays of magnetic and other dielectric components. Indeed, the presence of a magnetic constituent can eliminate the time reversal $R$ from the point symmetry group $G$ of the periodic array. The space inversion $I$, in turn, can always be removed by proper geometry of the periodic structure, even if each individual constituent is a centrosymmetric material. In this way, the macroscopic symmetry $G$ of a magnetic photonic crystal can always be made compatible with spectral asymmetry. Although such a composite does not display any static magnetoelectric effect, dynamically it can behave as an artificial bianisotropic medium with exceptionally strong electromagnetic spectral asymmetry, unachievable in any natural material.

Strong spectral asymmetry can result in the phenomenon of electromagnetic unidirectionality $[1,5]$. A unidirectional medium, being perfectly transparent for plane electromagnetic waves propagating in a certain direction, "freezes" the radiation of the same frequency propagating in the opposite direction. At the frozen mode frequency, the incident electromagnetic wave can be transmitted in the unidirectional photonic slab with little or even no reflection and completely converted into a coherent slow mode (the frozen mode) with drastically enhanced amplitude. Such a unique behavior can be very attractive for a variety of microwave and optical applications. This may include light amplification and lasing, enhancement of nonlinear effects used for higher harmonic generation and wave mixing, enhancement of the reciprocal and non-reciprocal optical activity, etc.

The rest of the paper is organized as follows. In the next section we take a closer look at the phenomenon of electromagnetic spectral asymmetry in magnetic photonic crystals and apply our approach to periodic layered structures incorporating magnetic layers. Then, we turn to the phenomenon of electromagnetic unidirectionality and the frozen mode regime. 


\section{GYROTROPIC PHOTONIC CRYSTALS}

At the frequency range of interest, all constitutive components of the photonic crystal are presumed electromagnetically lossless. As a consequence of spatial periodicity, the electromagnetic frequency spectrum of a photonic crystal develops a band-gap structure similar to that of electrons in a crystal lattice [6]. We assume that each of the constitutive components of photonic crystal is a uniform dielectric material satisfying conventional constitutive relations

$$
D=\varepsilon(\omega) \boldsymbol{E}, \quad \boldsymbol{B}=\mu(\omega) \boldsymbol{H}
$$

with Hermitian material tensors

$$
\varepsilon^{\dagger}(\omega)=\varepsilon(\omega), \mu^{\dagger}(\omega)=\mu(\omega)
$$

The dagger $\dagger$ indicates the Hermitian conjugate. The property (12) of Hermitivity implies electromagnetic losslessness of the medium. The tensors $\boldsymbol{\varepsilon}(\omega)$ and $\boldsymbol{\mu}(\omega)$ are different in different components of the periodic array. The absence of magnetoelectric terms in the constitutive relations (11) implies that each uniform component, if it fills the entire space, has perfectly symmetric electromagnetic dispersion relation $\omega(\boldsymbol{k})=\omega(-\boldsymbol{k})$, which is the case with all nonmagnetic and overwhelming majority of magnetic materials. At the same time we expect that spatially periodic array of such "non-magnetoelectric" components can support essentially asymmetric electromagnetic spectrum (4). In other words, in magnetic photonic crystals, the property (4) of bulk spectral asymmetry can be achieved by proper space arrangement of the constitutive components, rather than by incorporating magnetoelectric materials [1].

\section{$\underline{\text { Symmetry of Maxwell equations in photonic crystals }}$}

From symmetry standpoint, photonic crystals, being spatially periodic, can be viewed as artificial macroscopic crystals. Therefore, every photonic crystal can be assigned certain magnetic symmetry group $G$, which along with rotations, reflections, and translations may also include time reversal operation $R$ combined with some space transformations [3]. Knowing magnetic symmetry $G$ of the periodic array, one can apply the criterion (7) to find out whether or not one can expect asymmetric dispersion relation for a particular direction of the wave vector $\boldsymbol{k}$. This can only occur if the symmetry group $G$ is on the list of those compatible with linear magnetoelectric effect [3]. It does not mean, though, that the magnetic photonic crystal can display any static magnetoelectric effect.

At first sight, the problem of assigning magnetic symmetry group $G$ to a photonic crystal seems to be quite straightforward. Indeed, knowing the geometry of the periodic array and the symmetry $G_{i}{ }^{0}$ of each individual constitutive component, one can immediately obtain the exact magnetic symmetry $G^{0}$ of the photonic crystal. The so obtained symmetry group $G^{0}$ will be referred to as the true symmetry group of the photonic crystal. By definition, the periodic array is invariant under operations from the true symmetry group $G^{0}$. The important point, though, is that the symmetry of the Maxwell equations (8) with the constitutive relations (11) can be higher than $G^{0}$. Indeed, as far as electrodynamics is concerned, each constitutive component $i$ of the periodic array is represented by the respective material tensors $\varepsilon_{i}(\omega)$ and $\mu_{i}(\omega)$. The symmetry $G_{i}$ of the material tensors $\varepsilon_{i}(\omega)$ and $\mu_{i}(\omega)$ can be higher than the symmetry $G_{i}^{0}$ of the material itself. For 
instance, both $\boldsymbol{\varepsilon}_{i}(\omega)$ and $\boldsymbol{\mu}_{i}(\omega)$, being second rank tensors, are always centrosymmetric regardless of whether or not the material itself supports space inversion. In other words, the symmetry of the material relations (11) in some of the constitutive components of the photonic crystal can be higher than the point symmetry $G_{i}^{0}$ of the respective material. The symmetry group $G$ that describes the electrodynamics of the photonic crystal is defined as the symmetry of the Maxwell equations (8) with material relations (11). Hereinafter, the group $G$ will be referred to as the electromagnetic symmetry group. The above argument shows that the electromagnetic symmetry group $G$ of the photonic crystal can be higher compared to its true magnetic symmetry group $G^{0}$.

If indeed the electromagnetic symmetry group $G$ appears to be higher than the true symmetry group $G^{0}$, one can expect the situation where a particular effect, such as spectral asymmetry, is prohibited by $G$ but allowed by $G^{0}$. In such a case, this particular effect can occur, but if it does, it is associated exclusively with physical processes unaccounted for by the Maxwell equations (8) with the conventional constitutive relations (11). All such interactions and effects are presumed insignificant. They may include, but are not limited to: electrostriction and/or magnetostriction, space dispersion (e.g., reciprocal optical activity in chiral materials, if any), surface effects at the interfaces between different components of the photonic crystal, magnetoelectric effect in constitutive materials, if any. Hereinafter, we will focus exclusively on the robust bulk electrodynamic effects which are accounted for by the Maxwell equations (8) with the conventional constitutive relations (11). Thus, our symmetry consideration will be based on the electromagnetic symmetry group $G$, rather than on its subgroup $G^{0}$. Note that in most cases the two symmetries are simply identical $\left(G \equiv G^{0}\right)$. An example to the contrary is a photonic crystal with a chiral or a ferroelectric constitutive component.

A photonic crystal can display electromagnetic spectral asymmetry (4) only if its symmetry group $G$ includes neither time reversal nor space inversion. If none of the constitutive components of a photonic crystal supports any kind of spontaneous magnetic order, nor is an external magnetic field applied, then the photonic crystal certainly possesses time reversal symmetry $R$ and supports perfectly symmetric dispersion relation (2). Thus, asymmetric dispersion relation can be found exclusively in magnetic photonic crystals.

The distinguishing feature of the material tensors $\varepsilon(\omega)$ and $\mu(\omega)$ in magnetically polarized media is that both tensors are complex

$$
\text { In magnetic media: } \varepsilon^{*}(\omega)=\varepsilon(-\omega) \neq \varepsilon(\omega), \mu^{*}(\omega)=\mu(-\omega) \neq \mu(\omega) \text {, }
$$

where the star denotes complex conjugate. By contrast, in lossless nonmagnetic media the tensors $\boldsymbol{\varepsilon}(\omega)$ and $\boldsymbol{\mu}(\omega)$ are real and symmetric

In non-magnetic media: $\varepsilon^{*}(\omega)=\varepsilon(-\omega)=\varepsilon(\omega), \mu^{*}(\omega)=\mu(-\omega)=\mu(\omega)$.

In homogeneous media, the imaginary (skew-symmetric) parts of $\boldsymbol{\varepsilon}(\omega)$ and $\boldsymbol{\mu}(\omega)$ are responsible for the nonreciprocal effect of Faraday rotation, while in periodic heterogeneous media the same terms can also cause the effect of electromagnetic spectral asymmetry. In fact, the degree of electromagnetic spectral asymmetry is directly related to the magnitude of Faraday rotation in the magnetic constituent of the photonic crystal. Note that in the static limit, the material tensors $\boldsymbol{\varepsilon}(\omega)$ and $\boldsymbol{\mu}(\omega)$ become real and symmetric 


$$
\operatorname{Im}(\varepsilon)=\operatorname{Im}(\mu)=0, \text { as } \omega \rightarrow 0
$$

implying that all electromagnetic nonreciprocal effects vanish. By contrast, in natural magnetoelectric crystals, where the electromagnetic spectral asymmetry is associated with the tensor $\chi$ in Eq (9), the magnetoelectric effect persists even if $\omega \rightarrow 0$, although it is extremely small at all frequencies.

Unlike the situation with time reversal symmetry $R$, the space inversion $I$ is always supported by both material tensors $\boldsymbol{\varepsilon}(\omega)$ and $\boldsymbol{\mu}(\omega)$ in every uniform constitutive component of the composite structure, regardless of presence or absence of magnetic and/or electric polarization, chirality, etc. To remove space inversion from electromagnetic symmetry group $G$ of the periodic array and, thereby, to allow for electromagnetic spectral asymmetry, one should choose a proper spatial arrangement of the constitutive components. To put it differently, the structural geometry of the photonic crystal must be complex enough not to support space inversion.

To sum up, we can state that only magnetic photonic crystals with special geometry can support asymmetric electromagnetic dispersion relation (4). The criterion (7) is just a necessary condition for spectral asymmetry. Even if this condition is met, the effect of spectral asymmetry may appear to be negligible or even ruled out by physical reasons different from those imposed by magnetic symmetry. To find out if a photonic crystal satisfying the criterion (7) does display the electromagnetic spectral asymmetry, one has to go beyond the symmetry consideration and solve the Maxwell equations (8) in the heterogeneous medium. Several specific examples are considered below.

\section{Nonreciprocal Periodic Stacks}

Photonic crystals can have one-, two- or three-dimensional periodicity. One-dimensional photonic crystals are commonly referred to as periodic stacks, or multilayers. The symmetry arguments based on the criterion (7) for spectral asymmetry can be applied with equal ease to photonic crystals of any dimensionality. But if we want to go further and actually solve the Maxwell equations in the composite medium, then the case of one-dimensional periodicity is the most attractive. On the other hand, magnetic multilayers might be the most practical composites supporting strong electromagnetic spectral asymmetry. Therefore, in further consideration we will focus exclusively on periodic magnetic stacks. Extensive information on electrodynamics of magnetic photonic crystals, including magnetic stacks, can be found in Ref. [7].

Let us start with periodic stacks with just two different layers in a unit cell, as shown in the example in Fig. 1. The electromagnetic symmetry group $G$ of such a periodic array always supports space inversion symmetry with the center of inversion in the middle of each uniform layer. Therefore, a periodic stack composed of two alternating layers will never display electromagnetic spectral asymmetry, regardless of the materials of the layers. Let us reiterate that referring to the electromagnetic symmetry group $G$ rather than to the true magnetic symmetry group $G^{0}$ of the photonic crystal, we disregard those presumably insignificant effects which cannot be accounted for within the framework of time-harmonic Maxwell equation (8) with conventional constitutive relations (11). In many cases, though, the symmetry groups $G$ and $G^{0}$ are simply identical. 


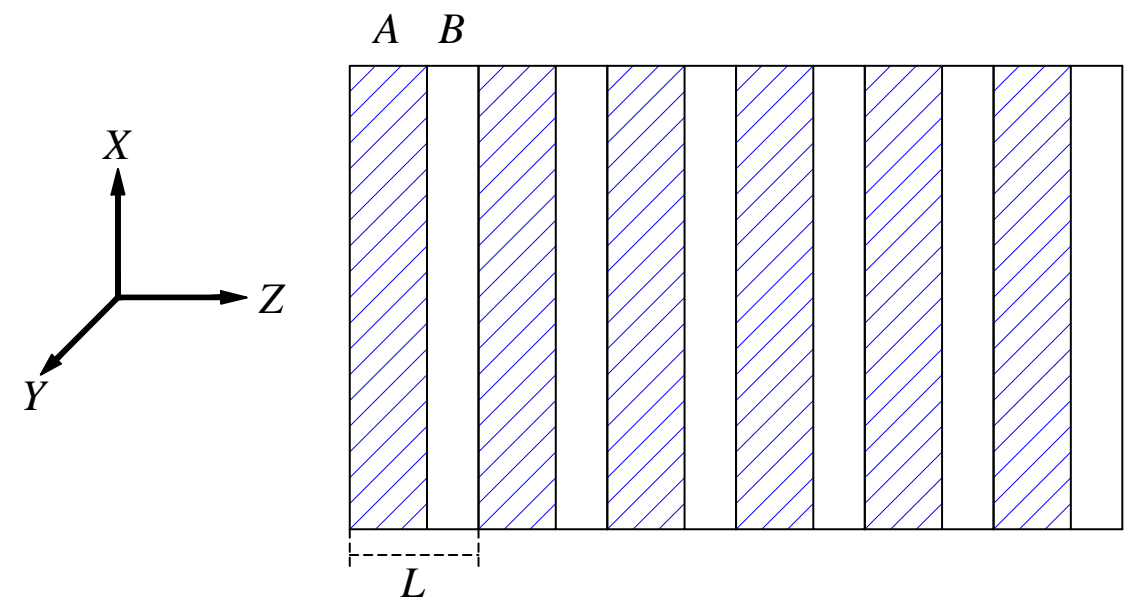

Figure 1. Periodic stack with two layers $A$ and $B$ in a unite cell $L$. This stack always supports symmetric dispersion relation (2), regardless of the material of the layers and the direction $\boldsymbol{k}$ of wave propagation.

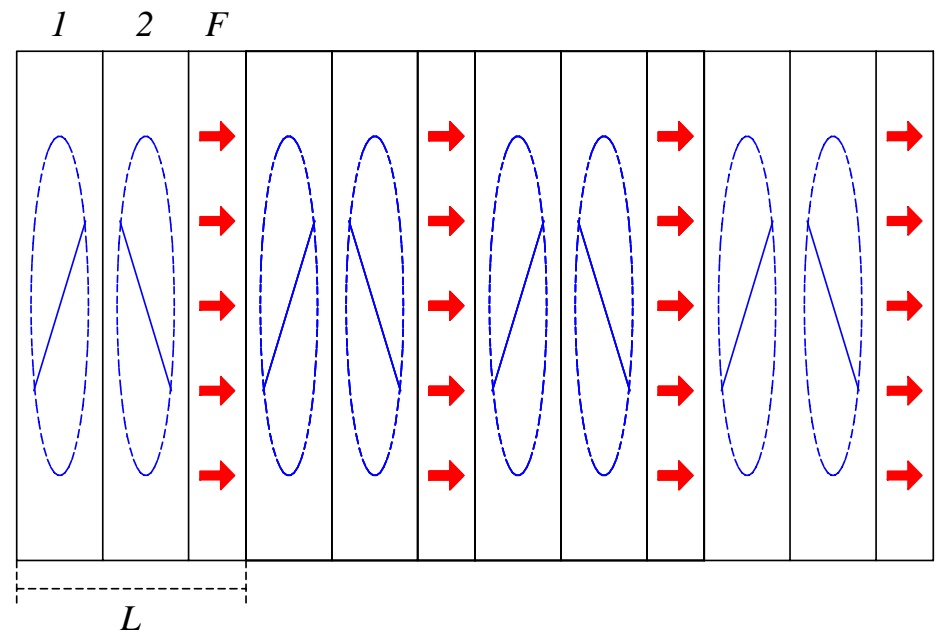

Figure 2. A simplest periodic magnetic stack capable of supporting asymmetric bulk dispersion relation (4). A unit cell $L$ of this stack comprises three layers: two anisotropic dielectric layers 1 and 2 with misaligned in-plain anisotropy (the $A$ - layers), and one magnetic layer $F$ with magnetization shown by the arrows.

Consider now periodic magnetic stacks with three layers in a unit cell. In this case there is a possibility of removing space inversion from the electromagnetic symmetry group $G$ of the periodic array. An example of how it can be done is shown in Fig. 2. The $F$ - layers in Fig. 2 are ferromagnetic with magnetization parallel to the $z$-direction normal to the layers. The respective material tensors are 


$$
\boldsymbol{\varepsilon}_{F}=\left[\begin{array}{ccc}
\varepsilon_{F} & i \alpha & 0 \\
-i \alpha & \varepsilon_{F} & 0 \\
0 & 0 & \varepsilon_{F}^{\prime}
\end{array}\right] ; \mu_{F}=\left[\begin{array}{ccc}
\mu_{F} & i \beta & 0 \\
-i \beta & \mu_{F} & 0 \\
0 & 0 & \mu_{F}^{\prime}
\end{array}\right]
$$

The real parameters $\alpha$ and $\beta$ are responsible for non-reciprocal circular birefringence (Faraday rotation). Both $\alpha$ and $\beta$ are odd functions of frequency $\omega$. At frequencies below $10^{12} \mathrm{~Hz}$, the dominant contribution to the Faraday rotation usually comes from the "magnetic" parameter $\beta$, which can become particularly large in the vicinity of a magnetic resonance.

For simplicity, the $A$ - layers are presumed nonmagnetic, although this is not a requirement. The respective permittivity and permeability tensors are

$$
\varepsilon_{A}=\left[\begin{array}{ccc}
\varepsilon_{A}+\delta \cos 2 \phi & \delta \sin 2 \phi & 0 \\
\delta \sin 2 \phi & \varepsilon_{A}-\delta \cos 2 \phi & 0 \\
0 & 0 & \varepsilon_{A}^{\prime}
\end{array}\right], \mu_{A}=\left[\begin{array}{ccc}
\mu_{A}+\Delta \cos 2 \phi & \Delta \sin 2 \phi & 0 \\
\Delta \sin 2 \phi & \mu_{A}-\Delta \cos 2 \phi & 0 \\
0 & 0 & \mu_{A}^{\prime}
\end{array}\right]
$$

The term "nonmagnetic" implies that both material tensors (14) are real and symmetric. Whether or not the magnetic permeability differs from unity has nothing to do with presence or absence of "magnetism" in terms of symmetry, because it does not affect the time reversal symmetry. The parameters $\delta$ and $\Delta$ describe the in-plane anisotropy, while the angle $\phi$ defines the orientation of the principle axes of the tensors $\varepsilon(\omega)$ and $\mu(\omega)$ in the $x-y$ plane. All $A$ - layers are made of the same anisotropic dielectric material and have the same thickness. The only parameter that may differ in different $A$ - layers is the orientation $\phi$.

The periodic array in Fig. 2 can display strong spectral asymmetry only if either of the two gyrotropic parameters $\alpha$ and $\beta$ in the magnetic constituent is large enough. Specifically, at least one of the two quantities $\alpha / \varepsilon_{F}$ or $\beta / \mu_{F}$ should be of the order of $10^{-1}$ or larger. Additional necessary condition for strong spectral asymmetry is that the in-plane dielectric anisotropy in the $A$ - layers is strong enough. Specifically, at least one of the two quantities $\delta / \varepsilon_{A}$ and/or $\Delta / \mu_{A}$ should be of the order of $10^{-1}$ or larger.

All essentially different periodic arrays of the $A$ - and $F$ - layers with three layers in a primitive cell are equivalent to a single array shown in Fig. 2. A primitive cell comprises one $F$ layer and two $A$ - layers with different orientations $\phi_{1}$ and $\phi_{2}$. The most critical parameter of this structure is the misalignment angle $\phi=\phi_{1}-\phi_{2}$ between the adjacent $A$ - layers. This angle determines the electromagnetic symmetry group $G$ of the stack, along with the symmetry of its electromagnetic dispersion relation. The results are summarized in the following table

Table 1.

$\begin{array}{ccc}\text { Misalignment angle } & \text { Magnetic symmetry } & \text { Spectral symmetry } \\ \phi=0 & m^{\prime} m^{\prime} m & \omega(\boldsymbol{k})=\omega(-\boldsymbol{k}) \text { for all } \boldsymbol{k} \\ \phi=\pi / 2 & \overline{4} m^{\prime} m^{\prime} & \omega(\boldsymbol{k})=\omega(-\boldsymbol{k}) \text { for } \boldsymbol{k} \square z \\ \phi \neq 0, \pi / 2 & 2^{\prime} 2^{\prime} 2 & \omega(\boldsymbol{k}) \neq \omega(-\boldsymbol{k}) \text { for } \boldsymbol{k} \square z\end{array}$


Note that in the case $\phi=0$, the three-layered unit cell in Fig. 2 reduces to a two layered cell with doubled thickness of the $A$ - layer. As we already know, the electromagnetic symmetry group of a periodic stack with two-layered unit cell always supports space inversion and, therefore, displays symmetric dispersion relation $\omega(\boldsymbol{k})=\omega(-\boldsymbol{k})$ for an arbitrary direction of the wave vector $\boldsymbol{k}$, regardless of the materials of the layers.

A typical numerical example of electromagnetic dispersion relations of the nonreciprocal periodic array in Fig. 2 is shown in Fig. 3. In accordance with the Table 1, the spectral asymmetry (4) for $\boldsymbol{k} \| z$ develops only if the misalignment angle $\phi$ is not a multiple of $\pi / 2$.

The above example presents the simplest and the most symmetric periodic stack supporting the bulk spectral asymmetry. More examples can be found in Ref. [1].
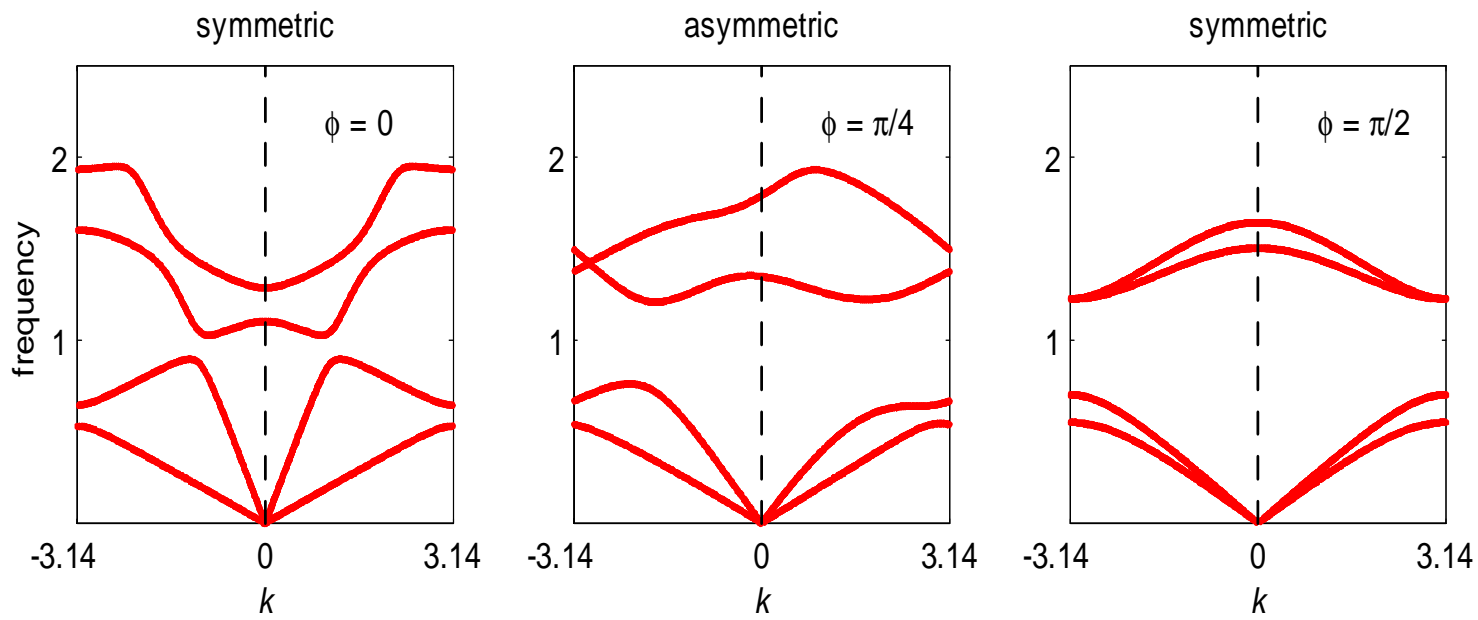

Figure 3. Example of electromagnetic dispersion relations $\omega(k)$ of the nonreciprocal periodic stack in Fig. 2. Three graphs correspond to three different values of the misalignment angle $\phi$ between adjacent $A$ - layers.

\section{ELECTROMAGNETIC UNIDIRECTIONALITY AND THE FROZEN MODE REGIME}

Strong electromagnetic spectral asymmetry has various physical consequences, one of which is the effect of unidirectional wave propagation. Suppose that at $k=k_{0}$ one of the spectral branches $\omega(\boldsymbol{k})$ develops a stationary inflection point

$$
\text { at } k=k_{0} \text { and } \omega=\omega_{0}=\omega\left(k_{0}\right): \quad \frac{\partial \omega}{\partial k}=0 ; \quad \frac{\partial^{2} \omega}{\partial k^{2}}=0 ; \quad \frac{\partial^{3} \omega}{\partial k^{3}} \neq 0 \text {, }
$$

as shown in Fig. $4 a$. With certain reservations, the energy velocity of electromagnetic wave coincides with its group velocity

$$
u(k)=\frac{\partial \omega}{\partial k} .
$$


At frequency $\omega=\omega_{0}$ there are two propagating Bloch waves: one with $k=k_{0}$ and the other with $k=k_{1}$. Obviously, only one of the two waves can transfer electromagnetic energy - the one with $k=k_{1}$ and the group velocity $u\left(k_{1}\right)<0$. The Bloch eigenmode with $k=k_{0}$ has zero group velocity $u\left(k_{0}\right)=0$ and does not transfer energy. This latter eigenmode is referred to as the frozen mode, it is associated with stationary inflection point (15) of the electromagnetic dispersion relation. As one can see in Fig. $4 a$, none of the two eigenmodes with $\omega=\omega_{0}$ has positive group velocity and, therefore, none of the electromagnetic eigenmodes can transfer energy from left to right at this particular frequency! Thus, a photonic crystal with the dispersion relation similar to that in Fig. $4 a$, displays the property of electromagnetic unidirectionality at $\omega=\omega_{0}$. Such a remarkable effect can be viewed as an extreme manifestation of the spectral asymmetry (4).

a) Dispersion relation

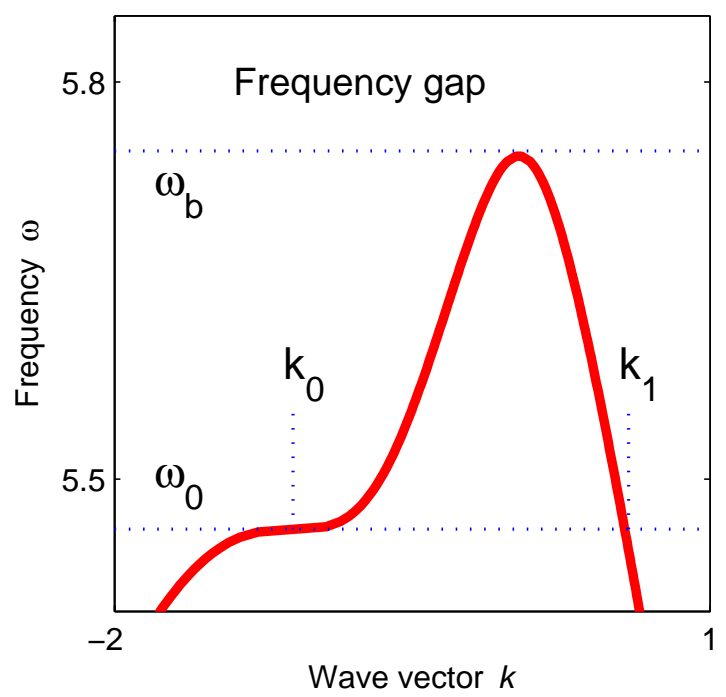

b) Transmittance

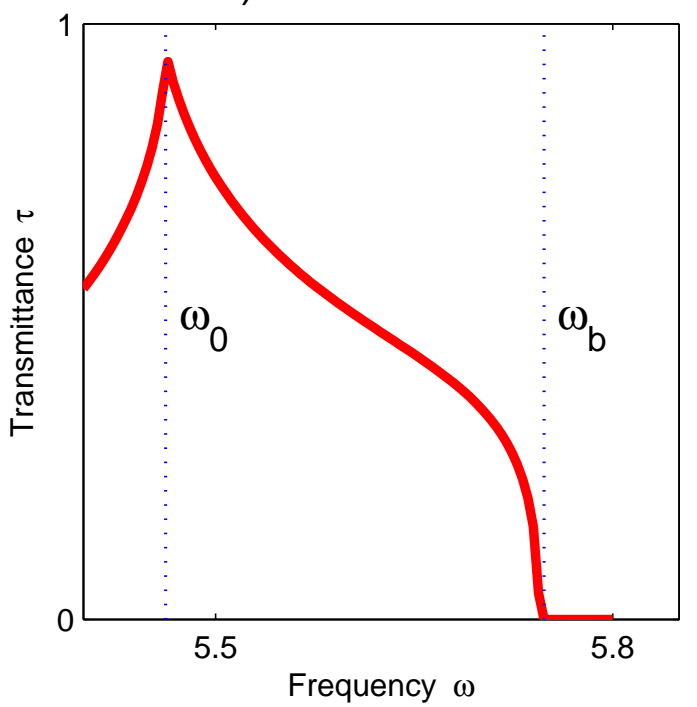

Figure 4. (a) A fragment of asymmetric dispersion relation $\omega(k)$ of the periodic stack shown in Fig. 2. At $k=k_{0}$ and $\omega=\omega_{0}$ this spectral branch develops a stationary inflection point associated with electromagnetic unidirectionality and the frozen mode. $\omega_{b}$ is the edge of the frequency band. (b) Frequency dependence of the transmittance $\tau$ of the semi-infinite photonic slab with the dispersion relation in Fig. $4 a$. The incident light propagates from left to right, as shown in Fig. 5. At the frequency $\omega_{0}$ of stationary inflection point, $\tau$ is close to unity, which implies that the incident wave is almost completely converted into the frozen mode with zero group velocity and drastically enhanced amplitude. The values of $\omega$ and $k$ are expressed in units of $c / L$ and $1 / L$, respectively.

The key physical conditions for the effect of electromagnetic unidirectionality are:

1) The electromagnetic symmetry group $G$ of the periodic array must be compatible with the criterion (7) for spectral asymmetry.

2) The magnetic constituent must display significant nonreciprocal circular birefringence at frequency range of interest (at least 10\%, or more).

3) The anisotropic layers must display significant in-plane anisotropy (at least 10\%, or more).

Failure to satisfy the conditions 2 and/or 3 does not formally rule out the phenomenon of electromagnetic unidirectionality, but it would obscure the effect. Specifically, weak Faraday rotation or weak anisotropy leads to a small value of the third derivative $\omega_{k}^{\prime \prime \prime}(k)$ in formula(15), 
which, in turn, pushes the stationary inflection point $\omega_{0}$ in Fig. $4 a$ too close to the photonic band edge $\omega_{b}$. In the close proximity of the photonic band edge, both forward and backward waves become equally slow. Besides, the photonic slab becomes totally reflective, which implies that the incident light cannot be transmitted in the photonic crystal and converted into the slow frozen mode.

\section{$\underline{\text { Electromagnetic properties of a semi-infinite unidirectional slab }}$}

Consider a plane electromagnetic wave incident on the boundary of a semi-infinite unidirectional photonic slab as shown in Fig. 5. The electromagnetic dispersion relation of the periodic array is shown in Fig. $4 a$. Due to spectral asymmetry of the photonic crystal, the case of the right-to-left incidence appears to be quite different and will not be discussed here. At the slab boundary, a portion of the incident wave is reflected back and the rest enters the semi-infinite slab. Let $S_{I}, S_{R}$, and $S_{T}$ be the energy flux of the incident, reflected and transmitted waves, respectively. Due to the energy conservation, $S_{I}+S_{R}=S_{T}$. The transmittance $\tau$ and reflectance $\rho$ of lossless semi-infinite slab are defined as

$$
\tau=\frac{S_{T}}{S_{I}}, \rho=-\frac{S_{R}}{S_{I}} .
$$

The energy conservation implies that $\rho=1-\tau$.

In the case of a single propagating mode, the transmitted energy flux $S_{T}$ inside the slab can be expressed in terms of the mode energy density $W_{T}$ and its group velocity (16)

$$
S_{T}=u(k) W_{T} .
$$

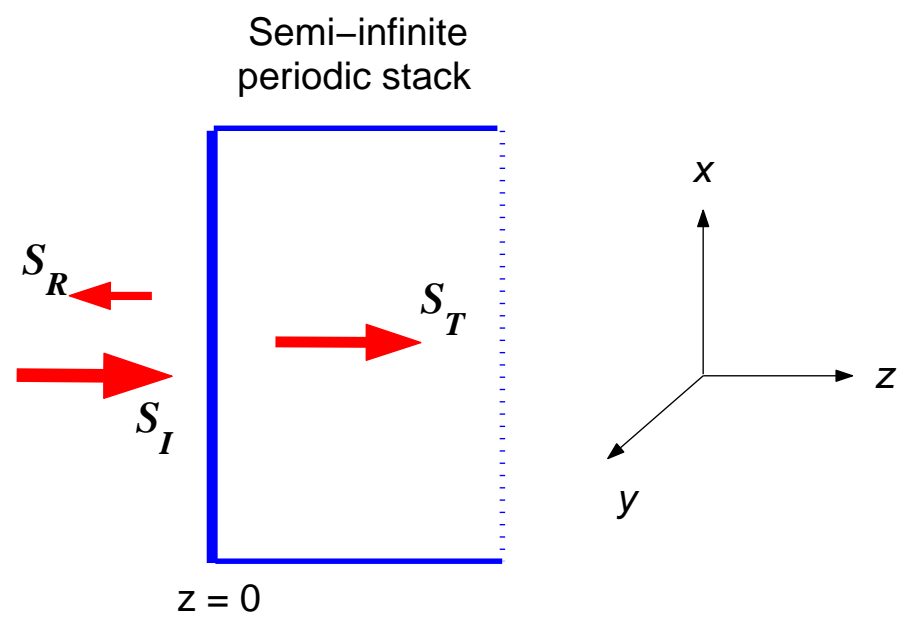

Figure 5. Plane electromagnetic wave normally incident on a semi-infinite photonic slab with the dispersion relation presented in Fig. $4 a$. The indices $I, R$, and $T$ indicate the incident, reflected and transmitted waves, respectively. 
According to Eq. (17) and Fig. 4a, the group velocity (16) of the transmitted wave vanishes as $\omega \rightarrow \omega_{0}$ and $k \rightarrow k_{0}$. At the same time, the transmittance $\tau$ along with the energy flux $S_{T}$ remain finite even at $\omega=\omega_{0}$, as seen in Fig. $4 b$. This implies that the electromagnetic energy density inside the unidirectional slab increases dramatically in the vicinity of the frozen mode frequency $\omega_{0}$

$$
W_{T} \square\left|\omega-\omega_{0}\right|^{-2 / 3} \text {, as } \omega \rightarrow \omega_{0} \text {, }
$$

while the wave slows down. The incident electromagnetic wave with frequency $\omega$ close to $\omega_{0}$ gets trapped inside the slab in the form of coherent frozen mode with drastically enhanced amplitude and nearly zero group velocity. In reality, the frozen mode amplitude will be limited by such factors as absorption, nonlinear effects, imperfection of the periodic array, deviation of the incident radiation from a perfect plane monochromatic wave, finiteness of the photonic slab dimensions, etc. Still, with all these limitations in place, the frozen mode regime can be very attractive for various practical applications. Detailed mathematical analysis of this remarkable phenomenon can be found in [8]. The frozen mode regime in nonmagnetic periodic stacks was considered in .

\section{ACKNOWLEDGMENTS}

The effort of A. Figotin and I. Vitebskiy was supported by the U.S. Air Force Office of Scientific Research under the grant FA9550-04-1-0359.

\section{REFERENCES}

1. A. Figotin, and I. Vitebsky. Phys. Rev. E63, 066609 (2001).

2. T. O'Dell. The Electrodynamics of Magnetoelectric Media (Amsterdam - North-Holland, 1970).

3. L. D. Landau, E. M. Lifshitz, L. P. Pitaevskii. Electrodynamics of continuous media. (Pergamon, N.Y. 1984)

4. A. Freeman and H. Schmid. Magnetoelectric Interaction Phenomena in Crystals. (New York: Gordon and Breach, 1979)

5. A. Figotin, and I. Vitebskiy. Phys. Rev. B67, 165210 (2003).

6. J. Joannopoulos, R. Meade, and J. Winn. Photonic Crystals. (Princeton University Press, 1995).

7. I. L. Lyubchanskii, N. N. Dadoenkova, et al. Phys. D: Appl. Phys. 36, R277(2003).

8. A. Figotin, and I. Vitebskiy. Phys. Rev. E 68, 036609 (2003). 